\title{
PHYSIOLOGICAL AND CELLULAR ASPECTS OF AGEING
}

A VARIETY of interesting papers were read at a joint meeting of the Dutch Society of Gerontology and the British Society for Research on Ageing, held at the Antoni van Leeuwenhoek-Huis (Netherlands Cancer Institute), Amsterdam, on July 27 and 28 . Included in the programme were papers by guest speakers from the United States, Canada and South Africa.

One of the difficulties in studying morphological or physiological changes with old age has been that of distinguishing between fundamental alterations due to senescence and changes due to the operation of secondary factors, for example, nutrition. Dr. J. J. Groen, of Amsterdam, described a condition, 'senile' paradentosis, which might have been thought at first to have been in either category. This condition is characterized by resorption of the alveolar bone of the tooth sockets, usually accompanied by retraction of the gingiva ; it is progressive and leads eventually to loss of teeth and to extensive atrophy of the jaw. The condition may begin in the young or middleaged. Another condition well known in old people is senile osteoporosis, particularly in the spine and pelvis. This condition is clinically recognizable by X-rays ; but it produces remarkably few metabolic changes. The phosphorus, calcium and phosphatase of serum of such patients seem to be unchanged, and the cause of the condition has been the subject of much speculation. Until recently, osteoporosis has been recorded by physicians and paradentosis by dentists-but Dr. Groen believes that they are one and the same disease. His investigations showed that all those suffering from one of them were found upon investigation to be suffering also from the other.

The etiology of this complaint was investigated at some length ; but only one constant factor could be found-in all cases the paivients were people who had stopped taking milk at an early stage in their lives. Investigation of their diets, which seemed adequate in most nutrients, showed a calcium intake becween 250 and $450 \mathrm{mgm}$. per day. A few patients on whom balance studies were done while they were on their 'home diet' had negative calcium balances of 50 to about $200 \mathrm{mgm}$. a day. Control individuals who drank a pint to a quart of milk a day showed scarcely any sign of paradentosis or of osteoporosis at an advanced age.

Dr. Groen pointed out that Allbright had thought that osteoporosis in old persons was due to a decrease in sex hormones; but his own investigations had shown that the disease can occur before the menopause and is invariably associated with a longprotracted low intake of calcium. He believes, in fact, that the condition 'paradentosis-osteoporosis' is mainly a result of a chronic, low-grade calcium deficiency.

Dr. T. Gillman, of South Africa, also recorded, the syndromes of many nutritional conditions met with in South Africa among native populations. These included kwashiorkor, pellagra and an accumulation of iron in the liver which was sometimes followed or accompanied by fibrosis; he stressed the significance of his results in the study of gerontology.
Clinical studies on blood pressure by Dr. M. Landowne, of Bethesda, using intra-arterial recording, showed that the systolic pressure increases by an average of $4.8 \mathrm{~mm}$. of mercury or 4 per cent per decade. This is the momentary peak pressure; but the average increase of the entire systolic period is $2.8 \mathrm{~mm}$. mercury or 2 per cent per decade. There is also a decreased cardiac output of $l$ per cent $p \in r$ year due partly to decrease in the blood pumped by each beat. Portion of this decrease is effected by a slowing of the heart. Just as in training for athletic activities the heart-rate slows, so in old age it slows as an adaptive change, for by so doing there is a large diastolic filling period, thus providing a better stroke/volume than if the diastolic pressure was shortened.

Dr. R. Van Zonneveld, of Gröningen, described a survey of old persons in the Netherlands which began with an inquiry among the old people themselves as to how they felt and what infirmities they com. plained of. This is to be followed by an announcement of the details of a survey which will be carried out in collaboration with twenty-four regional teams of doctors.

The question of whether sex hormones are invariably decreased in old age was discussed by Dr. $O$. Mühlbock, of the Netherlands Cancer Institute, on the basis of his work on highly inbred mice. Frequently in old female mice an enormous hypertrophy of the uteri is observed. It was found that the œstro. genic hormones maintaining this uterine development are produced by the ovaries. These old ovaries show few follicles; but cells can be seen in the periphery, obviously coming from the germinal epithelium, which look very like granulosa cells. They penetrate into the ovarian stroma forming small anovular follicles which are probably the source of at least some of the female sex hormone. In some cases the adrenals too may contribute ostrogens in old mice. In old adrenals so-called ' $A$ ' cells develop just under the cortex. These cell groups may show adenomatous development, and in this case probably also produce ostrogens.

In some animals there is also an enormous development of the mammary glands. This may be in part due to female sex hormones, but also to the high production of prolactin by the old pituitary. It was found that these mice often had pituitary tumours of a similar nature to those which can be produced in younger animals by prolonged ostrogen treatment. These pituitary tumours contain no gonadotrophin, growth hormone or corticotropin, and only a little thyrotrophic hormone. A high prolactin activity is, however, regularly found.

These studies emphasized that hormone imbalance may occur in old age, leading to endocrine hyperactivity rather than inactivity in some cases, and that this appears to be genetically determined.

Kidney studies of old rats by Dr. and Mrs. Fried. man, of Vancouver, also demonstrated endoerine changes in old animals. In this case it is apparently decreased neurohypophysial function which is responsible for their results. They found that both young and old animals appear to excrete sodium 
identically, but that the older animals excrete a much more watery urine which suggests a decrease of the tubular water-absorption mechanism. If 'Pitressin' is given to the old animals with the saline solutions used in producing a salt load on the kidneys, re-absorption of water becomes the same as in the young animals, although sodium re-absorption was a little less. The neurohypophysis was shown to be unresponsive to direct osmotic stimulus in the old rat.

Cellular and tissue studies in old age were described by Dr. P. J. Thung, of the Netherlands Cancer Institute, Dr. G. H. Bourne, of London, and Dr. H. Saxl, of the Department of Medicine, Leeds.

Dr. Thung's paper dealt with amyloidosis in senile mice. He pointed out that mice easily develop amyloid, which may be produced experimentally in response to injection of a wide variety of substances and even by a milk and cheese diet. In old age, however, untreated mice similarly often develop amyloid. Genetical factors appear to be of prime importance in this tendency to amyloid degeneration, both experimentally and spontaneously, as some strains are rarely affected whereas others may show a 100 per cent incidence. Many of the substances which in mice. cause amyloid, in the rat produce only necrosis and fibrosis. Similarly, it was found that chronic alcoholism, which in man would be expected to lead to liver cirrhosis, in mice caused heavy degrees of amyloidosis of the liver.

In human pathology it is increasingly recognized that amyloid degeneration is far more frequent than the classical condition found secondary to chronic suppurative disease. Its various forms range from this secondary amyloidosis to the amyloid found in cases of rheumatoid arthritis and the amyloid infiltrations frequently observed in the cardiac muscle in old age. These forms apparently represent different varieties of a common derangement of protein metabolism, on the causes of which as yet little is known. In mice, however, this derangement occurs much more easily than in man, and the causative agents consequently are less specific. Among these, old age plays an important part.

Dr. G. H. Bourne described the principal changes that take place in cells as they age, and directed attention to the fact that in many organs in old animals there is a histochemical increase in the activity of phosphatases and esterases and that biochemical studies have recorded an increase in activity of other hydrolytic enzymes. It may be that this increase in hydrolytic enzyme activity, which is destructive of many esters essential in metabolic activities, is one of the fundamental changes taking place in ageing cells.

The question of a possible link between protein and polysaccharide involvement in arteriosclerosis and lipid mesabolism was discussed by Dr. H. Saxl, of the Department of Medicine, Leeds. She suggested the following mechanism by which the tissue poly. saccharides in situ and certain components of the serum form an anti-lipæmic system: $(a)$ in the presence of elastase, and especially the fraction which appears to have elasto-mucase activity, the mucopolysaccharides are made soluble and unstable; (b) the action of the serum inhibitor is to inhibit the removal of the soluble polysaccharides and by a direct action on these to make them stable and orthochromatic, and either secondarily or as a result of this interaction to activate a lipase.
An examination of the mode of the lipolytic action on atheromatous arterial walls has shown that there appear to be a degradation of neutral lipids of the atheromatous plaques and liberation of the soluble acid fats, which diffuse in the tissues.

G. H. BOURNE

\section{CHEMISORPTION SYMPOSIUM AT KEELE}

A SYMPOSIUM on chemisorption was held by the Chemical Society during July 16-19 in the University College of North Staffordshire, Keele. The symposium, which was organized by Prof. W. E. Garner, was divided into five sections, the first of which dealt with the theory of chemisorption, with Prof. Garner as chairman. D. A. Dowden (Billingham) gave a general review of the covalent aspects of adsorption, extending the usual classification of $s p$ and $d$ bonds from metals to semiconductors. $\mathrm{H}_{\Theta}$ considered that adsorbed gases could either be held strongly on the intermetallic type of $d s p$ orbitals, or more weakly on atomic $d$ orbitals. Using the ideas of crystal field theory, he indicated how the $d^{3} s p^{2}$ orbitals of a surface metal atom might split to give a $d^{2} p$ set for the underlying metal and a $d s p$ orbital in the surface. He did not consider that the surface metal atoms exhibit $d s p$ free valencies, but emphasized the role of $d z$ orbitals in weak adsorption. T. B. Grimley (Liverpool) used the L.C.A.O. or tightbinding approximation to discuss the interaction of a hydrogen atom with an electron in a surface state of the metal. Where a singly occupied surface state or partly occupied $d$-band is available, a bond is to be expected, the hydrogen atom being located over the metal atom. J. H. de Boer (Geleen) gave a critical account of theories relating heat of adsorption to surface coverage, and contact potential. He considered particularly the cases of cæsium on tungsten and hydrogen on nickel. The deficiencies of the simple double-layer theory were described and then improvements, allowing for discrete layers and mutual depolarization of dipoles, were tried. The conclusion reached was that some heterogeneity was present even with cæsium on tungsten. G. C. A. Schuit, L. L. van Reijen and N. H. de Boer (Amsterdam) described thermodynamic data for the adsorption of hydrogen on nickel supported on silica, and other supported transition metals. Definite evidence for heterogeneity was deduced, and experiments on the hydrogen-deuterium exchange showed the existence of a few sites specially active at $77^{\circ} \mathrm{K}$. In the discussion, the thought was expressed that the silica support might in some way be contributing to the heterogeneity observed.

The next section dealt with chemisorption on insulators, and the chair was taken by D. A. Dowden. J. J. Kipling and D. B. Peakall (Hull) described results on the adsorption of liquids on silica gel and alumina. Four tests were made to distinguish chemisorption from physisorption: irreversibility; time effects; evidence for reaction at specific sites; and the relation of extent of chemisorption to the number and nature of active groups in the surface. S. J. Gregg (Exeter) described how some water was retained by active oxides even at the highest temperatures, probably hydroxyl groups. A second veriety of adsorption, probably involving molecular 